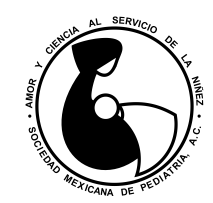

ARTí́CULO ORIGINAL

Vol. 86, No. 4 • Julio-Agosto 2019

pp 138-142

Revista Mexicana de

\title{
Asociación entre función motora gruesa y desnutrición en niños con parálisis cerebral que acuden a un centro de rehabilitación
}

\author{
Giussepe Doménico Pérez-Moya, ${ }^{1, *}$ Ana Alicia Ochoa-Lares, ${ }^{2}$ Sandybell González-Lugo ${ }^{3}$ \\ ${ }^{1}$ Médico Pediatra; ${ }^{2}$ Nutrióloga; ${ }^{3}$ Investigadora adjunta. Centro de Rehabilitación e Inclusión Infantil Teletón (CRIT), \\ Hermosillo, Sonora, México.
}

\begin{abstract}
RESUMEN
Introducción: La parálisis cerebral (PC) es la discapacidad física más común en los niños. Se ha descrito que los niños con PC presentan algún grado de desnutrición; sin embargo, hay poca información al respecto. Objetivo: Determinar la prevalencia de desnutrición en niños con PC que acuden al Centro de Rehabilitación e Inclusión Infantil Teletón (CRIT), Sonora, en el periodo 2016-2017, y su asociación con la función motora gruesa. Material y métodos: Es un estudio transversal. Con la autorización del comité de ética, se analizó una muestra no probabilística de 180 pacientes con PC, a los cuales, previo consentimiento informado, se les realizó antropometría y se obtuvo información del expediente. Se estableció un diagnóstico nutricional mediante curvas específicas para población con PC, así como por los criterios de Waterlow. Posteriormente se clasificaron en dos grupos, uno con sujetos con movilidad poco limitada (GMFCS nivel I-III) y otro de sujetos con movilidad gravemente limitada (GMFCS nivel IV-V). Para la comparación se utilizó Chicuadrada. Resultados: La prevalencia de desnutrición fue de $57.2 \%(n=103)$. Mientras que el porcentaje de desnutrición en los niños con movilidad gravemente limitada fue de $65.2 \%$, en comparación al $44.1 \%$ en el grupo con movilidad poco limitada $(p<0.05)$. Conclusiones: La desnutrición en niños con PC es frecuente y se incrementa en quienes tienen mayor limitación de la función motora.
\end{abstract}

Palabras clave: Parálisis cerebral, función motora gruesa, desnutrición, prevalencia.

\begin{abstract}
Introduction: Cerebral palsy (CP) is the most common physical disability in children. It has been described that children with CP have some degree of malnutrition; however, the information is limited. Objective: To determine the prevalence of undernutrition in children with $\mathrm{CP}$ attending Rehabilitation Center and Children Inclusion Telethon (CRIT), Sonora, in the period from 2016 to 2017 , and its association with gross motor function. Material and methods: This is a cross-sectional study. With authorization of the ethics committee, a non-probabilistic sample of 180 patients with CP was analyzed, to whom, prior informed consent, anthropometry was performed and information was obtained from the clinical file. With a specific curve for the population with PC and the Waterlow criteria, a nutritional diagnosis was established in each patient. Subsequently, the sample was grouped into two, one of subjects with limited mobility (GMFCS level I-III) and another of subjects with severely limited mobility (GMFCS level IV-V). Statistical analysis was performed with Chi-square. Results: The prevalence of undernutrition was $57.2 \%(n=103)$. While the percentage of undernutrition in children with severely limited mobility was $65.2 \%$ compared to $44.1 \%$ in the group with little mobility limited $(p<0.05)$. Conclusions: Undernutrition in children with $\mathrm{CP}$ is common and is higher in those with more limited motor function.
\end{abstract}

Keywords: Cerebral palsy, gross motor function, malnutrition, prevalence.

*Correspondencia: GDPM, pediatra.gperez@gmail.com

Conflicto de intereses: Los autores declaran que no tienen.

Citar como: Pérez-Moya GD, Ochoa-Lares AA, González-Lugo S. Asociación entre función motora gruesa y desnutrición en niños con parálisis cerebral que acuden a un centro de rehabilitación. Rev Mex Pediatr. 2019; 86(4):138-142.

[Association of gross motor function and undernutrition in children with cerebral palsy attending to a rehabilitation center] 


\section{INTRODUCCIÓN}

La parálisis cerebral (PC) es la discapacidad física más común en los niños. Se define como un grupo de afecciones motoras, cognitivas y perceptivas secundarias a una lesión cerebral no progresiva que afecta al cerebro en desarrollo. ${ }^{1}$ Se estima que, a nivel mundial, ocurre en dos a 2.5 casos por cada 1,000 recién nacidos vivos. ${ }^{1}$ En México, el 2\% de los jóvenes (15-29 años) y $1.6 \%$ de los niños (0-14 años) presenta algún tipo de discapacidad; sin embargo, se desconoce cuántos de estos tienen PC. ${ }^{2}$

El estudio de las condiciones nutricionales de los niños con PC tiene problemas, debido a la falta de un método estandarizado para dicha evaluación, así como tablas o curvas de referencia apropiadas. Se ha demostrado que el peso esperado para la talla es un indicador poco útil del estado nutricional de los niños con PC, por lo que se ha recomendado la medición de pliegues cutáneos, pero el uso de estas mediciones puede ser difícil. ${ }^{3}$

En general, los profesionales de la salud utilizan las curvas de crecimiento como herramienta para monitorizar cómo es que un niño gana peso y talla, comparado con un niño de la misma edad y sexo, tanto para identificar aquéllos que pierden peso o detienen su crecimiento, como para identificar candidatos a realizar estudios y recibir tratamiento para revertir esta condición. ${ }^{4}$ Estos conceptos pudieran ser aplicados para niños con PC; sin embargo, no crecen ni ganan peso al igual que sus pares sanos; incluso, entre los mismos niños con PC existe diferente grado de afección pondoestatural. ${ }^{4}$

Debido a que las curvas de crecimiento habituales están limitadas para su uso e interpretación en niños con discapacidad neuromotora, ${ }^{3}$ se desarrollaron curvas específicas para niños y niñas de dos a 20 años, tomando

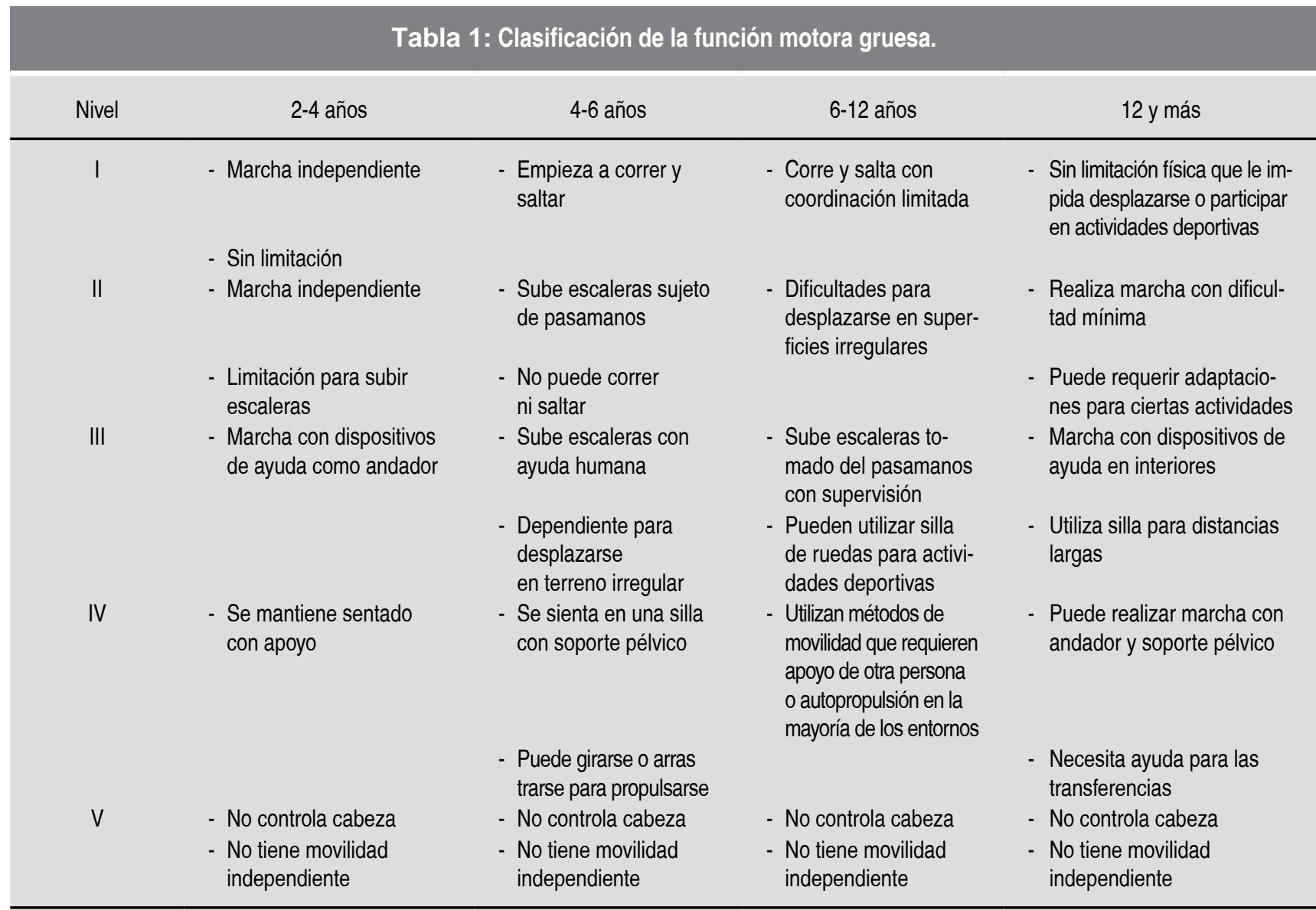

Fuente: Palisano R, Rosenbaum P, Walter S, et al. Development and reliability of a system, to classify gross motor function in children with cerebral palsy. Dev Med Child Neurol. 1997, 39: 214-223. 


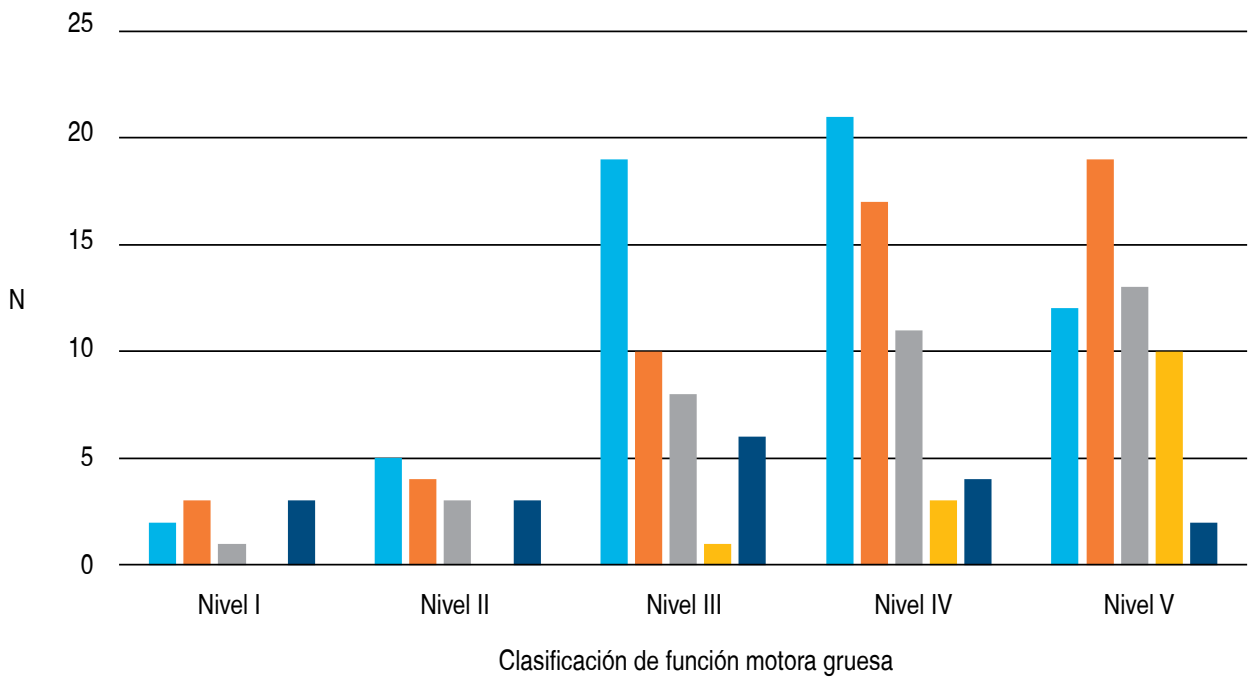

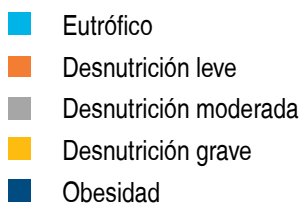

Figura 1:

Distribución del estado nutricional por nivel funcional en pacientes con parálisis cerebral. en cuenta cada uno de los cinco niveles del sistema de clasificación de la función motora gruesa (GMFCS, por sus siglas en inglés) (Tabla 1), ${ }^{5}$ siendo el nivel V el que tiene mayor discapacidad. Posteriormente, estos niveles se estratificaron de acuerdo a si son alimentados o no por gastrostomía; por lo anterior, existen 12 curvas de referencia, las cuales, además incluyen el riesgo de mortalidad dado por la condición nutricia. ${ }^{5}$ De esta forma, el propósito de estas curvas es identificar de manera objetiva si un paciente con $\mathrm{PC}$ tiene desnutrición, a fin de evitar su sobrediagnóstico y, en consecuencia, la sobrealimentación. Conviene señalar que estas curvas se realizaron de forma inicial en California, pero ya han sido validadas en otras poblaciones, identificando los mismos patrones de crecimiento. ${ }^{4}$

El estado nutricional tiene un impacto significativo en la salud y calidad de vida de los niños con PC. La doble carga de la malnutrición (obesidad y desnutrición) generalmente tiende a aumentar la necesidad de cuidados relacionados con la salud, y reduce su participación en actividades sociales y educacionales. Además, la malnutrición frecuentemente se asocia con deficiente circulación periférica y cicatrización, así como aumento en la espasticidad e irritabilidad. ${ }^{6}$

Cuando la lesión cerebral se presenta en la región frontal-insular en la vía de los ganglios basales, afecta la deglución, generando disfagia,${ }^{1}$ que se manifiesta como dificultad para la alimentación, como tiempos prolongados de alimentación, salivación excesiva, tos, episodios de atragantamiento al momento de la deglución, historial de neumonías aspirativas y en consecuencia desnutrición. ${ }^{3}$ Se ha reportado que la disfagia afecta desde 19 a 99\% de los niños con PC, ${ }^{6}$ observándose que es mayor en niños con cuadriplejía que cuando presentan diplejía. ${ }^{7}$ Se ha demostrado que la disfagia es un factor importante que afecta la condición nutricia de los niños con PC. ${ }^{8,9}$

Se conoce que la malnutrición afecta de 46 a $90 \%$ de los niños con PC. ${ }^{3}$ En México, existe poca información sobre la condición nutricia de estos niños, ${ }^{10}$ por lo que los objetivos del presente estudio fueron describir la prevalencia de desnutrición en los niños con PC atendidos en el CRIT Sonora, además de identificar la relación que existe entre el nivel función motora y el estado nutricional.

\section{MATERIAL Y MÉTODOS}

El estudio se realizó en el CRIT Sonora, que brinda atención a niños con discapacidad desde el nacimiento hasta los 17 años 11 meses de edad.

\section{Muestra}

Durante el periodo de estudio, la población de sujetos con PC atendidos en CRIT Sonora fue de 251 niños, de los cuales cumplieron los criterios de selección 180. Se incluyeron pacientes de ambos sexos, de dos a 19 años de edad, cuyos padres aceptaron participar mediante la carta de consentimiento informado y que en el expediente clínico la información sobre la manera de alimentación estuviera completa.

\section{Metodología}

Una sola persona realizó antropometría a los sujetos seleccionados. Para medir peso se utilizó una bás- 
cula de plataforma para silla de ruedas (Medidata WCS400/800), pesando primero la silla de ruedas para después tarar la báscula y pesar al sujeto sobre la silla, la misma báscula se usó cuando los pacientes podían lograr la bipedestación. Para medir la talla se utilizó una cinta métrica (Seca 201). La medición de la estatura se realizó por segmentos en quienes no lograron la bipedestación o presentan contracturas. En cada paciente se calculó el índice de masa corporal (IMC) y se contrastaron los datos obtenidos con las curvas específicas para sexo y nivel de GMFCS y, para clasificar el estado de nutrición, se utilizó la clasificación de Waterlow.

Análisis estadístico. Toda la información se capturó en una hoja de cálculo para su posterior análisis estadístico en SPSS versión 20.0. Las variables cualitativas se presentan como frecuencias simples y porcentajes, y las cuantitativas como promedio y desviación típica.

\section{RESULTADOS}

De los 180 sujetos incluidos, el 42.7\% $(\mathrm{n}=77)$ correspondió a mujeres y $57.3 \%(\mathrm{n}=103)$ a hombres. La distribución de acuerdo con el nivel funcional mostró nueve sujetos (5.0\%) con GMFCS nivel I, 15 (8.3\%) con GMFCS nivel II, 44 (24.4\%) en nivel III, 56 (31.1\%) en nivel IV y, por último, 56 (31.1\%) con nivel V.

De acuerdo con el estado nutricional hubo 59 pacientes (32.7\%) eutróficos, 53 (29.4\%) con desnutrición leve, 36 (20.0\%) con desnutrición moderada y 14 (7.7\%) con desnutrición grave; además, encontramos 12 (6.6\%) sujetos con sobrepeso y seis (3.3\%) con obesidad.

En la Figura 1 se muestra la distribución del estado de nutrición de acuerdo con el grado de afección de la función motora; como se observa, existe un mayor número de pacientes con desnutrición conforme aumenta el grado de afección. Sin embargo, al realizar el análisis estadístico no se encontró diferencia significativa $(\mathrm{p}=$ 0.059), pero al comparar a los pacientes con limitaciones leves para la movilidad (nivel I, II y III) con quienes tenían limitaciones graves para la movilidad (niveles IV y V), se encontró mayor prevalencia de desnutrición en este último grupo (Tabla 2), siendo estadísticamente significativo $(\mathrm{p}=0.005)$.

Por otro lado, cuando se contrastó la consistencia de alimento ingerido con el estado de nutrición, hubo una mayor proporción de pacientes alimentados con papillas que tenían desnutrición grave, en comparación con pacientes alimentados con picados o de consistencia normal, lo cual resultó estadísticamente significativo $(\mathrm{p}<0.001)($ Tabla 3).

\section{DISCUSIÓN}

De acuerdo con estos resultados, el estado nutricional parece estar relacionado con el nivel funcional en niños con PC. Sin embargo, esta asociación solamente se logró observar al agrupar el nivel funcional en dos, con mayor (nivel IV y V de GMFCS) y menor nivel de función motora (niveles I al III de GMFCS), lo que concuerda con lo descrito en la bibliografía internacional. ${ }^{8}$

La Sociedad Europea de Gastroenterología y Hepatología Pediátrica (ESPGHAN), en su guía para tratamiento y evaluación nutricional de los niños con discapacidad neurológica, no recomienda el uso de las curvas de crecimiento específicas para parálisis cerebral; no obstante, la fuerza de la recomendación es débil, ${ }^{3}$ lo que abre la puerta a que dichas curvas sean utilizadas a criterio del clínico. Por nuestra parte, consideramos que es mejor utilizar estas curvas, ya que además de ayudar a hacer el diagnóstico del estado de nutrición permiten predecir con el riesgo de morbilidad y mortalidad relacionado con el estado nutricional. ${ }^{4}$

En cuanto a la prevalencia de desnutrición, se observó en nuestro grupo de estudio que $57 \%(\mathrm{n}=103)$ de los sujetos presenta algún grado de desnutrición, hallazgos similares a lo descrito en otro estudio con niños mexicanos, reportando una prevalencia de 49\%; sin embargo, se utilizaron curvas de crecimiento para población general a diferencia del nuestro, donde se utilizaron las curvas específicas para nivel funcional y sexo. ${ }^{10}$

Se observó una asociación entre la consistencia de los alimentos y el estado nutricional, resultando mayor

Tabla 2: Comparación del estado de nutrición

de acuerdo con el grado de afección de función motora en niños con parálisis cerebral $(\mathrm{N}=180)$.

\begin{tabular}{|c|c|c|c|}
\hline & \multicolumn{2}{|c|}{ GMFCS } & \multirow[b]{2}{*}{$\underset{0.0056^{*}}{p}$} \\
\hline & $\begin{array}{c}\text { Nivel I-III } \\
(n=68) \\
n(\%)\end{array}$ & $\begin{array}{l}\text { Nivel IV-V } \\
(n=112) \\
n(\%)\end{array}$ & \\
\hline Desnutrición & $30(44.1)$ & 73 (65.2) & \\
\hline $\begin{array}{l}\text { No desnutrición } \\
\text { (eutróficos + } \\
\text { sobrepeso/obe- } \\
\text { sidad) }\end{array}$ & $38(55.9)$ & $39(34.8)$ & \\
\hline
\end{tabular}


Tabla 3: Estado de nutrición de acuerdo con la consistencia del alimento ingerido en niños con parálisis cerebral.

\begin{tabular}{|c|c|c|c|c|c|c|c|c|}
\hline \multirow[b]{3}{*}{ Estado nutricional } & \multirow[b]{3}{*}{$\mathrm{N}$} & \multicolumn{5}{|c|}{ Consistencia del alimento } & & \multirow{3}{*}{$\begin{array}{c}p \\
0.0001^{*}\end{array}$} \\
\hline & & \multicolumn{2}{|c|}{ Normal } & \multicolumn{2}{|c|}{ Picado fino } & \multicolumn{2}{|c|}{ Papilla } & \\
\hline & & $n$ & $\%$ & $\mathrm{n}$ & $\%$ & $\mathrm{n}$ & $\%$ & \\
\hline Eutrófico & 59 & 45 & 76.3 & 10 & 16.9 & 4 & 6.8 & \\
\hline Desnutrición I & 53 & 31 & 58.5 & 16 & 30.2 & 6 & 11.3 & \\
\hline Desnutrición II & 36 & 16 & 44.4 & 13 & 36.1 & 7 & 19.4 & \\
\hline Desnutrición III & 14 & 4 & 28.6 & 2 & 14.3 & 8 & 57.1 & \\
\hline Sobrepeso & 12 & 10 & 83.3 & & & 2 & 16.7 & \\
\hline Obesidad & 6 & 5 & 83.3 & 1 & 16.7 & & & \\
\hline Total & 180 & 111 & & 42 & & 27 & & \\
\hline
\end{tabular}

prevalencia de desnutrición en los sujetos alimentados con papillas. En otros estudios se ha encontrado una relación directa entre la prevalencia de disfunción oromotora y la desnutrición; ${ }^{8}$ en nuestro estudio no se interrogó acerca de datos de disfunción oromotora. Se ha reportado que la disfunción oromotora afecta hasta $80 \%$ de los pacientes con GMFCS nivel IV-V, por lo que podrían tener más riesgo de desnutrición. ${ }^{8}$

Resulta relevante hablar sobre el tipo de alimentación, ya que se ha reportado que pacientes con desnutrición y son alimentados con dieta polimérica de forma exclusiva mediante gastrostomía, mejoran el peso, IMC y el pliegue tricipital. ${ }^{11}$

\section{CONCLUSIONES}

En niños con parálisis cerebral, entre mayor es la alteración de la función motora se incrementa la desnutrición, lo cual puede relacionarse con el tipo de alimentación que se ofrece a estos pacientes. Esta información deberá servir para ajustar las recomendaciones de alimentación de acuerdo a las características de cada paciente.

\section{REFERENCIAS}

1. Erasmus CE, Van Hulst K, Rotteveel JJ, Willemsen MAAP, Jongerius $\mathrm{PH}$. Swallowing problems in cerebral palsy. Eur $J$ Pediatr. 2012; 171: 409-414.

2. Las personas con discapacidad en México: una visión al 2010. Instituto Nacional de Estadística y Geografía. México: INEGI, 2013.
3. Romano C, Van Wynckel M, Hulst J, Broekaert I, Bronsky J, Dall'Oglio Let-al. European Society for Paediatric Gastroenterology, Hepatology and Nutrition Guidelines for the evaluation and treatment of gastrointestinal and nutritional complications in children with neurological impairment. J Pediatr Gastroenterol Nutr. 2017; 65(2): 242-264.

4. Wright CM, Reynolds L, Ingram E, Cole TJ, Brooks J. Validation of US cerebral palsy growth charts using a UK cohort. Dev Med Child Neurol. 2017; 59(9): 933-938.

5. Palisano R, Rosenbaum P, Walter S, Russell D, Wood E, Galuppi B. Development and reliability of a system, to classify gross motor function in children with cerebral palsy. Dev Med Child Neurol. 1997; 39: 214-223.

6. Penagini F, Mameli C, Fabiano V, Brunetti D, Dilillo D, Zuccotti GV. Dietary intakes and nutritional issues in neurologically impaired children. Nutrients. 2015; 7: 9400-9415.

7. Hariprasad PG, Elizabeth KE, Valamparampil MJ, Kalpana $D$, Anish TS. Multiple nutritional deficiencies in cerebral palsy compounding physical and functional impairments. Indian J Palliat Care. 2017; 23: 387-392.

8. Melunovic M, Hadzagic-Catibusic F, Bilalovic V, Rahmanovic S, Dizdar S. Anthropometric parameters of nutritional status in children with cerebral palsy. Mater Sociomed. 2017; 29(1): 68-72.

9. García-Contreras AA, Vásquez-Garibay EM, RomeroVelarde E, Ibarra-Gutiérrez Al, Troyo-Sanromán R. Energy expenditure in children with cerebral palsy and moderate/ severe malnutrition during nutritional recovery. Nutr Hosp. 2015; 31(5): 2062-2069.

10. Vega-Sanchez R, Gomez-Aguilar ML, Haua K, Rozada G. Weight-based nutritional diagnosis of Mexican children and adolescents with neuromotor disabilities. BMC Research Notes. 2012; 5: 218.

11. Dipasquale V, Catena MA, Cardile S, Romano C. Standard polymeric formula tube feeding in neurologically impaired children: a five-year retrospective study. Nutrients. 2018; 10: 684. 